\title{
Optimization Design of Ultra-low Frequency Electromagnetic Transmitter Coil
}

\author{
Ji Xiaoxu ${ }^{1, a}$, Zhang Tao ${ }^{1, b^{*}}$ Yan Yining ${ }^{1, c}$ and Mo Qingkai ${ }^{1, d}$ \\ School of Manufacturing Science and Engineering, Sichuan University, Chengdu \\ a434719563@qq.com, bnic6700@scu.edu.cn, c719670143@qq.com, d921255070@qq.com
}

\begin{abstract}
Keywords: ultra-low frequency; electromagnetic transmission; bifilar; optimization design
Abstract. Ultra-low frequency electromagnetic transmitter is typically used equipment that tracking position in the pipeline. Effective receiving distance which is determined by the emission field strength is one of the most important performance parameters of such equipment. Moreover, transmitting antenna coil parameters have a decisive effect on the emission field strength. We conclude by theoretical calculation between emission field strength and coil parameters. Under existing conditions, such as battery, core, and volume, the bifilar can exponentially increase the effective radiated magnetic field strength; improve the performance of the penetration distance. Then, we experimentally verify the effectiveness of this optimization.
\end{abstract}

\section{Foreword}

In the process of oil and gas pipelines regular maintenance and cleaning, pigs in the pipelines must be tracked and located in the whole process. Reasonable pipeline maintenance and operating procedures can greatly improve the life of the pipeline and eliminate security risks in the production process. The proper tracking measures and technologies can confirm the main pipeline failure clogged locations to promptly eliminate clogging fault. Otherwise, it will cause serious consequences with pipeline efficiency drops even scrapped.

Currently, most pig configuration of tracking system is based on the principle of ultra-low electromagnetic pulse transmitting and receiving. Its working principle is: the tracking system is fixed to the pig, and the transmitter coil emits ultra-low frequency electromagnetic pulse. The receiver of surface receives the signal through the probe receiving coil, and tracking the position by the processing identification of the pig. To ensure the electromagnetic pulse can effectively penetrate metal pipes and soil rock, the frequency is around $23 \mathrm{~Hz}$.

Because emission field strength is needed to be large enough (is proportional to the effective receiving distance), so transmitter coil generally use high permeability perm alloy material as the core, while increasing coil winding turns to increase its inductance, which to some extent to meet the emission field strength requirements. But generally it remains within the volume limits law generally single coil wound caused by poor performance parameters cannot launch a large magnetic field strength, so that which effect the problem of effective reception distance shortage. The design on the condition that the existing battery, the core and allowed volume, and by theoretical calculation of the relationship between effective radiated field strength and the coil parameters, obtained using bifilar winding can exponentially increase the effective radiated field strength and improve penetration distance performance findings. Its effectiveness of the proposed optimization scheme is verified by experimental results, which will undoubtedly have a strong inventiveness and practical significance in engineering applications.

\section{Transmit coil optimization design calculation}

\section{Magnetic antenna transmission system}

VLF transmitter system uses a special kind of "antenna"; its transmitter calculation methods are different with general radio transmitters.

(1)Spatial magnetic field of the energized solenoid 
As we all know, the magnetic field generated by the solenoid in an energized state, whether the solenoid has core or not, are based on the rotating body in the solenoid axis as a rotation axis of symmetry. Its cross-sectional view of magnetic field lines distribution as shown in Fig.1. In the direction of the solenoid axis, the magnetic field strength is the strongest. The transmitter can send pig position signal, which is rely on such an alternating magnetic field in the space transmission.

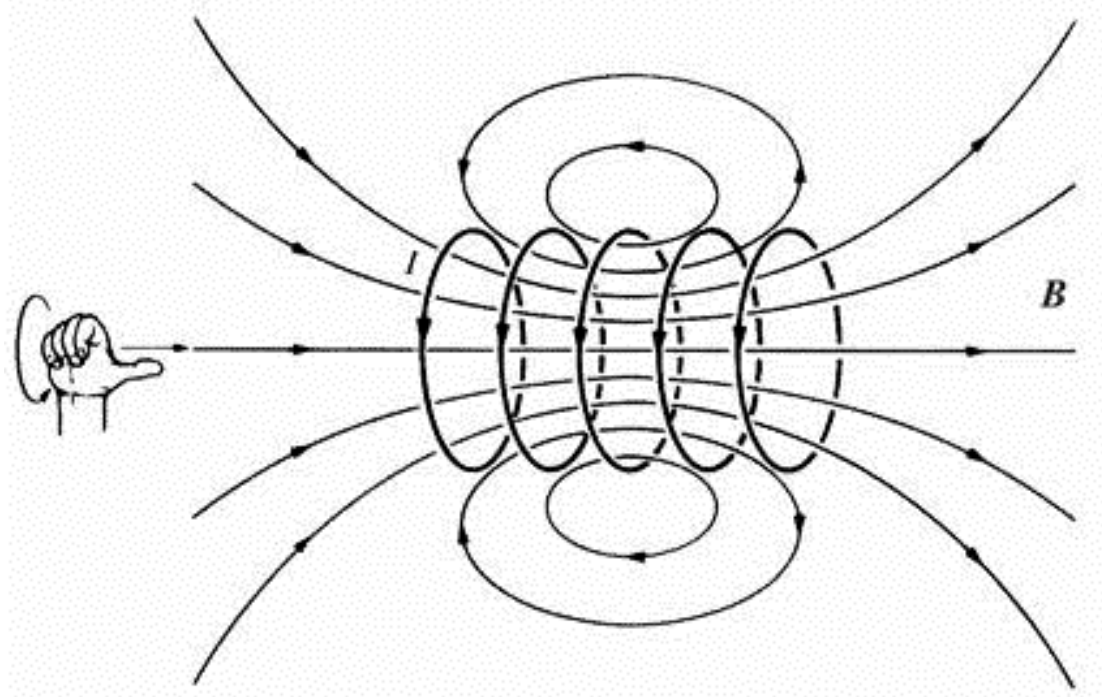

Fig. 1 solenoid magnetic field space

(2) The spatial distribution of the magnetic field strength $\mathrm{H}$

To deduce the situation of solenoid external magnetic field distribution, we make two assumptions:

(a) Relative permeability of the solenoid core $\mu_{r}$ is large enough (more than 104 actually), the magnetic core loss is zero, that is, assuming that the core of the solenoid wire on a flat surface, like a magnetic dipole.

(b) If a multilayer circular coil and a single circular coils energize the same current (same NI), produce same magnetic field strength at the center of the circle, then the single circular coil diameter is called equivalent diameter of the multilayer circular coil denoted $D_{z}$. The coil magnetic field we studied is a single layer coil diameter $D_{z}$ that the magnetic field generated.

After the above assumptions, we'll have a core that has a certain axial length and a certain radial thickness of the multilayer solenoid coil reduces to a no length and no thickness plane single coil namely magnetic dipole.

The book "Electromagnetic Field and Wave" shows a magnetic dipole field distribution relationship: $\bar{H}=\frac{1}{\mu_{0}} \bar{\nabla} \times \bar{A}=\frac{m_{0}}{4 \pi r \overline{\lambda^{2}}} e^{j \omega t}\left[2\left(\frac{\overline{\lambda^{2}}}{r^{2}}+j \frac{\bar{\lambda}}{r}\right) \cos \theta \overline{r_{1}}+\left(\frac{\overline{\lambda^{2}}}{r^{2}}+j \frac{\bar{\lambda}}{r}-1\right) \sin \theta \overline{\theta_{1}}\right]$

When $\bar{\lambda} \gg r$, space vector $\bar{H}$ (amplitudes of $\bar{H}$ at different locations on the space) can be calculated: $\bar{H}=\frac{m_{0}}{4 \pi r^{3}}\left(2 \cos \theta \overline{r_{1}}+\sin \theta \overline{\theta_{1}}\right)$

In the formula: $m_{0}-$ Magnetic dipole moment, $m_{0}=\sqrt{2} I N \pi R^{2} ; \overline{r_{1}}$ - in the direction of $r$ unit vector; $\overline{\theta_{1}}$ - in the direction of $\theta$ unit vector; $\mathrm{N}$ - coil turns; I - current effective value; R- coil equivalent radius, $\mathrm{R}=\frac{D_{Z}}{2}, \bar{\lambda}$ - Radian length, $\lambda$ - wavelength, $\bar{\lambda}=\frac{\lambda}{2 \pi}$.

$r, \theta$ and ${ }^{\varphi}$ are three parameters spherical coordinate system. It is shown in Fig. 2. Let $\mathrm{P}(\mathrm{x}, \mathrm{y}, \mathrm{z})$ of the point in space, so that the point $\mathrm{P}$ can also be determined by three orderly numbers $r, \theta$ and $\varphi$. And $\mathrm{r}$ is 
the distance between the origin $\mathrm{O}$ and the point $\mathrm{P} . \theta$ is an angle between positive $\mathrm{z}$-axis and the directed line segment. And $\varphi$ is an angle from the positive $\mathrm{x}$-axis in the counterclockwise direction to go directed line segment by the positive z-axis view. Such three numbers $r, \theta, \varphi$ called the spherical coordinates of the point $\mathrm{P}$. Range of variation for $r, \theta, \varphi$ is $0 \leq r<+\infty, 0 \leq \emptyset \leq \pi$, $0 \leq \theta \leq \pi, r=$ constant, scilicet with the origin as the heart of the sphere; $\theta=$ constant, scilicet the origin is the vertex, $\mathrm{z}$ axis by a conical surface; ${ }^{\varphi}=$ constant, scilicet over the half-plane $\mathrm{x}$-axis; Among them, $\mathrm{x}=\cos \varphi, \mathrm{y}=r \sin \theta \sin \varphi, \mathrm{z}=r \cos \theta$

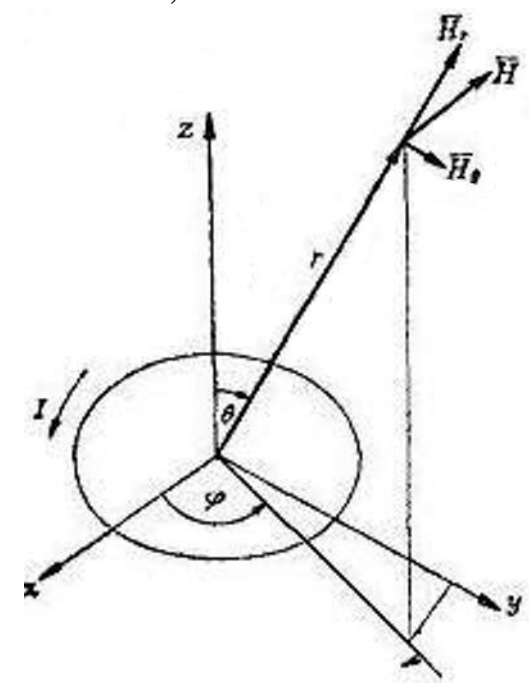

Fig. 2 magnetic dipole field intensity $\bar{H}$ distributions

Objective to study the transmitter antenna is to increase the magnetic field strength of the coil magnetic field and increase the distance signal transmission for effectively penetrate the soil, rock and pipeline. It can be seen by the equation (1) and

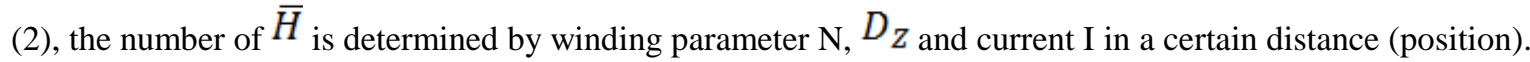
$I=\frac{U_{L}}{\omega L}=\frac{\sqrt{2}(U-\Delta U)}{\omega L}$

In the formula: L - Coil inductance; $U_{L}$ - Coil terminal voltage; $\omega$ - Angular frequency; U - Power voltage; $\Delta \mathrm{U}$. Transistor saturation voltage drop.

The formula (3) shows that when the coil terminal voltage $U$ and angular frequency are determined, current I depend on the inductance of the coil L. L is depend on magnetic material (core), coil turns, geometry and shape of the magnetic circuit. In order to find ways to improve the strength of the coil magnetic field, it is necessary to calculate the transmitter coil inductance.

\section{The calculation of magnetic core multilayer solenoid inductors}

Tab. 1 shape factor $\mathrm{K}$ values

\begin{tabular}{ccccccccccccccc}
\hline$\frac{2 R}{l_{T}}$ & 0.1 & 0.2 & 0.3 & 0.4 & 0.6 & 0.8 & 1.0 & 1.5 & 2.0 & 3.0 & 4.0 & 5.0 & 10 & 20 \\
\hline $\mathrm{K}$ & 0.96 & 0.92 & 0.88 & 0.85 & 0.79 & 0.74 & 0.69 & 0.60 & 0.52 & 0.43 & 0.37 & 0.32 & 0.20 & 0.12 \\
\hline
\end{tabular}




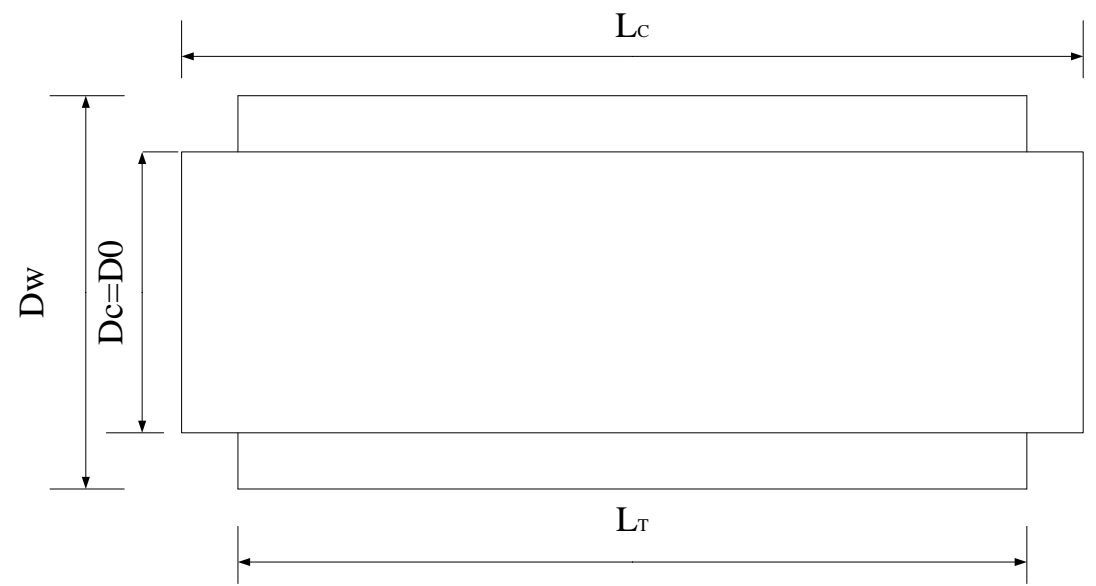

$D_{W}$ - The outer diameter of the coil; $D_{O}$ - The inner diameter of the coil; $D_{C}$ - The outer diameter of the core; $L_{T}$ - Coil length; $L_{C}$ - Core length

Fig. 3 sectional view of the core multi-solenoid

Since the core is a magnetic equipotent surface in a same cross-section (surface composed of equal magnetic potential points), thus the entire cross section have same magnetic field strength which is equal with the magnetic field strength at the center of the circle. As a result, the inner and outer diameters of the core multilayer solenoid becomes $D_{O+} D_{C}$ and $D_{W+} D_{C}$ (Sectional view of the core multi-solenoid shown in Fig. 3). In the coil winded by research group that $D_{C} \approx D_{O}$.

The same with the calculation method of $\bar{L}_{0}$, for the magnetic flux in the core multi-solenoid we deduced the following formula:

$\Phi=K_{D} \bullet \frac{\sqrt{2}}{4} \mu_{0} N I \pi D_{d}$

In the formula: $K_{D}$ - Solenoid ends coefficient; $\mathrm{N}$ - Solenoid coil turns; $D_{d}$ - Core solenoid magnetic moment equivalent diameter.

Thus it can be obtained:

$L=\frac{K N \phi}{\sqrt{2} I}=\frac{1}{4} K_{D} K \mu_{0} N^{2} \pi D_{d}$

The formula:

$$
K_{D}=\frac{2 D_{z}^{2}}{\left[1+\frac{2 n(2 n+1) d^{2}}{3 D_{\omega} D_{0}}\right] D_{\omega} D_{0}} \text {. }
$$

And the equivalent diameter when calculated according to the magnetic with core:

$D_{d}=\sqrt{2}\left(1+\frac{D_{c}}{2 D_{\omega}}\right)\left[1+\frac{n(2 n+1) d^{2}}{3 D_{\omega} D_{0}}\right] \sqrt{D_{\omega} D_{0}}$

Substituted into the formula $\mathrm{L}$ and simplifying it which can be obtained: 


$$
\begin{aligned}
& L=\frac{\sqrt{2}}{2} K \mu_{0} N^{2} \pi\left(1+\frac{D_{c}}{2 D_{\omega}}\right) \bullet \frac{D_{z}^{2}}{\left[1+\frac{n(2 n+1) d^{2}}{3 D_{\omega} D_{0}}\right] \sqrt{D_{\omega} D_{c}}} \cdot \frac{l_{c}}{l_{T}} \\
& \approx \frac{\sqrt{2}}{2} K \mu_{0} N^{2} \pi\left(1+\frac{D_{c}}{2 D_{\omega}}\right) D \cdot \frac{l_{c}}{l_{T}} \\
& \approx \frac{\sqrt{2}}{2} K \mu_{0} N^{2} \pi\left(1+\frac{D_{c}}{2 D_{\omega}}\right) D_{z} \cdot \frac{l_{c}}{l_{T}} H
\end{aligned}
$$

In the Transmitter coil:

$$
l_{T}=151 \mathrm{~mm}, l_{c}=130 \mathrm{~mm}, D_{c} \approx D_{0}=18 \mathrm{~mm}, \quad D_{\omega}=18+\frac{8250}{289} \times 0.41 \times 2=41.41 \mathrm{~mm} \frac{2 R}{l_{T}}=\frac{18}{151}=0.1
$$

Query Tab. 1 draw shape factor $\mathrm{K}=0.96$.Permeability of vacuum $\mu_{0}=4 \pi \times 10^{-7} \mathrm{H} / \mathrm{m}$,

$\mathrm{N}=8250$. Multi-coil magnetic moment equivalent diameter:

$$
D_{z}=\frac{D_{\omega}-D_{0}}{\ln \frac{D_{\omega}}{D_{0}}}=\frac{23.41}{\ln 2.3}=28.11 \mathrm{~mm}
$$

$L=0.96 \times 4 \pi \times 10^{-7} \times 8250^{2} \times \pi\left(1+\frac{18}{2 \times 41.41}\right) \times 28.11 \times 10^{-3} \times \frac{130}{151}=23.87 \mathrm{H}$.

By $f_{0}=\frac{1}{2 \pi \sqrt{L C}}=23 H z$, we obtained that: $C=\left(\frac{1}{2 \pi f_{0}}\right)^{2} / L=2 \mu f$

\section{The magnetic field strength of the transmitter}

The $H_{Z}$ is the amplitude of the magnetic field strength of the transmitter antenna coil axis that can be calculated using the following formula:

$\overline{H_{z}}=\frac{m_{0}}{4 \pi r^{3}}\left(2 \cos \theta \overline{r_{1}}+\sin \theta \overline{\theta_{1}}\right)=\frac{m_{0}}{2 \pi z^{3}} \bullet \bar{z}$

(The axial direction, $\theta=0, \overline{r_{1}}=\bar{z}, \cos \theta=1, \sin \theta=0$ )

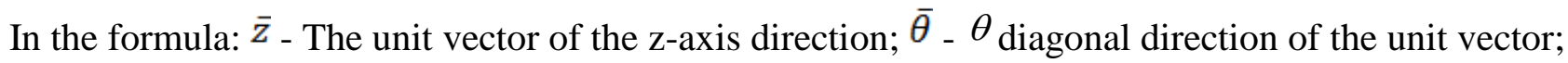
$\overline{r_{1}}$ - The radius $\mathbf{r}$ of the unit direction vector. And $H_{Z}$ is calculated using the formula:

$$
\begin{aligned}
& H_{z}=\frac{m_{0}}{2 \pi z^{3}}=\frac{\sqrt{2} N_{1} I \pi D_{d}^{2}}{8 \pi z^{3}} \\
& =\frac{\sqrt{2}\left(1+\frac{D_{c}}{2 D_{w}}\right)\left[1+\frac{n(2 n+1)}{3 D_{w} D_{0}}\right] \sqrt{D_{w} D_{c}}(U-\Delta U) d_{1}}{2 K \mu_{0} \pi \omega(n+1) l_{c} z^{3}} .
\end{aligned}
$$

( $\omega$ - Angular frequency, $d_{1}$ - Wire diameter, $\mathrm{U}$ - Power voltage, $\Delta \mathrm{U}$ - Transistor saturation voltage drop, $\mathrm{n}$ - Coil layers)

It can be concluded that the possible measures to improve emission intensity of the magnetic field are: 1. Increase the diameter of the coil $d_{1}$, reducing coil layers $n$, namely to reduce the number of turns of wound coil $N_{1}$, enhanced magnetic field strength. Therefore, if the coil winding manner from the previous single wire wound improves to double wire wound, it can increase the strength of the magnetic field of the transmitter coil double to the original.

2. Increase $D_{C}$, shorten $l_{c}$, it can enhance the magnetic field strength of transmission system. 
Increasing the power supply voltage can be proportionally increasing the magnetic field strength of transmission system. Limited by existing battery, core and allowed volume conditions, measures of 2 and 3 can not be adopted. Measure 1 is feasible.

Formula (11) shows that $\mathrm{A}$ is a function of the distance $\mathrm{z}$. In order to facilitate comparison that magnetic field strength of the transmitter coils in each case, we require the magnetic field strength $H_{1}$ at $1 \mathrm{~m}$ is $\mathrm{z}$, as a measure of the magnetic field strength standard of the transmitter coil. Larger the value of $H_{1}$, the magnetic field coil is stronger.

Original transmitter coil is a single-wound, and put parameters in $H_{1}, \mathrm{U}=12 \mathrm{~V} \mathrm{~V}$, $l_{c}=130 \mathrm{~mm}, D_{c} \approx D_{0}=18 \mathrm{~mm}, D_{w}=41.41 \mathrm{~mm}, d_{1}$ - diameter of the wire.

$$
\begin{aligned}
& H_{1 s}=\frac{\sqrt{2}\left(1+\frac{D_{c}}{2 D_{w}}\right)\left[1+\frac{n(2 n+1) d_{1}^{2}}{3 D_{w} D_{0}}\right] \sqrt{D_{w} D_{c}}(U-\Delta U) d_{1} \times 10^{-3}}{2 K \mu_{0} \pi \omega(n+1) l_{c}} \\
& =\frac{\sqrt{2}\left(1+\frac{18}{2 \times 41.11}\right)\left[1+\frac{29 \times(2 \times 29+1) \times 0.41^{2}}{3 \times 41.41 \times 18}\right] \times \sqrt{41.41 \times 18} \times(12-0.3) \times 0.41 \times 10^{-3}}{2 \times 0.96 \times 4 \pi \times 10^{-7} \times \pi \times 2 \pi \times 23 \times(29+1) \times 130}
\end{aligned}
$$

$=0.054 \mathrm{~A} / \mathrm{m}$.

The improved transmit coil is double-wound.

$\mathrm{U}=12, l_{c}=130 \mathrm{~mm}, D_{c} \approx D_{0}=18 \mathrm{~mm}, D_{w}=41.41 \mathrm{~mm}, d_{1}=0.82 \mathrm{~mm}$

$$
\begin{aligned}
& H_{1 s}=\frac{\sqrt{2}\left(1+\frac{D_{c}}{2 D_{w}}\right)\left[1+\frac{n(2 n+1) d_{1}^{2}}{3 D_{w} D_{0}}\right] \sqrt{D_{w} D_{c}}(U-\Delta U) d_{1} \times 10^{-3}}{2 K \mu_{0} \pi \omega(n+1) l_{c}} \mathrm{~A} / \mathrm{m} \\
& =\frac{\sqrt{2}\left(1+\frac{18}{2 \times 41.11}\right)\left[1+\frac{29 \times(2 \times 29+1) \times 0.41^{2}}{3 \times 41.41 \times 18}\right] \times \sqrt{41.41 \times 18} \times(12-0.3) \times 0.82 \times 10^{-3}}{2 \times 0.96 \times 4 \pi \times 10^{-7} \times \pi \times 2 \pi \times 23 \times(29+1) \times 130}
\end{aligned}
$$

$=0.108 \mathrm{~A} / \mathrm{m}$.

\section{Experimental researches}

The measurement method of the transmitter effective transmission range is place the transmitter in a fixed position and turn on the transmitter. Receiver moves away along a direction parallel to the transmitter axis. And we constantly observe the amplitude of the received signal by the receiver. When receiving the signal amplitude is reduced to about $1 / 3$ of the initial signal amplitude intensity, namely that the receiver can not receive effectively. The distance measured by long-term soft feet at this time between the transmitter and the receiver is the transmitter effective emission distance. The effective emission distance experimental data from the transmitter before and after improvements as shown in Tab. 2 and Tab. 3.

Tab. 2 the transmitter effective emission distance before improvement

\begin{tabular}{lllllllllll}
\hline $\begin{array}{l}\text { Experiment } \\
\text { No }\end{array}$ & 1 & 2 & 3 & 4 & 5 & 6 & 7 & 8 & 9 & 10 \\
\hline $\begin{array}{c}\text { Distance } \\
(\mathrm{m})\end{array}$ & 8.2 & 8.5 & 9.2 & 8.7 & 8.6 & 8.8 & 8.3 & 9.0 & 8.7 & 8.6 \\
\hline
\end{tabular}


Tab. 3 the transmitter effective emission distance after improvement

\begin{tabular}{lllllllllll}
\hline $\begin{array}{l}\text { Experiment } \\
\text { No }\end{array}$ & 1 & 2 & 3 & 4 & 5 & 6 & 7 & 8 & 9 & 10 \\
\hline $\begin{array}{c}\text { Distance } \\
(\mathrm{m})\end{array}$ & 15.8 & 15.9 & 16.3 & 16.2 & 16.1 & 16.5 & 16.4 & 16.9 & 16.5 & 16.2 \\
\hline
\end{tabular}

As we can see from the above experimental data: The original single-wound system effective transmission range is about 9 meters. Improved dual-wound system effective transmission range is about 16 meters. It can be seen the improved dual-wound system without changing the original transmitter system battery, the core and volume, the effective transmission range almost doubled.

\section{Conclusions}

We conclude by theoretical calculation between emission field strength and coil parameters. Under existing conditions, such as battery, core, and volume, the bifilar can exponentially increase the effective radiated magnetic field strength; improve the performance of the penetration distance. The conclusions actually applied to an existing transmitter system, the effective transmission range has increased from $9 \mathrm{~m}$ to $16 \mathrm{~m}$. It confirmed the effective feasibility of this optimization.

\section{Acknowledgements}

This project was supported by National Science and Technology Support Plan (2015BAF27B00) and Sichuan Provincial Science and Technology Support Plan (2013GZX0154-3,2015GZ0163, 2015GZ0034, 2016GZ0023, 2016GZ0184).

\section{References}

[1] Qingshuang Zhang. Pig valve applications in the petroleum and natural gas industry [J]. General Machinery, 2007, (Z1):73-78.

[2] Linzhang Wang, Meiji Zhang, Jinsheng Zhang. VLF transmitter study of pig tracker[J]. Gas Storage and Transportation, 1983(6):16-39.

[3] Dongquan Kuang, Gong Jing. Study on Cleaning Pipes by Electronic Location [J]. Journal of Shenyang Polytechnic University, 1996, 18(2):104-108.

[4] Hongjun Chen, Xiaohua Zhang, Junyuan Li. Ultra Low Frequency Electromagnetic Wave Localization and Application to Pipeline Robot [J]. International Conference on Intelligent Robots and Systems. Beijing, China, 2006:1201-1205.

[5] Huaguang Kang, Shoubin Zou. Basis of Electronic Technology digital part [M]. Higher Education Press, Beijing, 2000.

[6] Li Gang, Qiaozhen Li, Hongjun Sun. 51 MCU Application of power saving mode [J]. Manufacturing Automation, 2000, 22(11):57-60. 